\title{
WA-GPSR: Weight-Aware GPSR-Based Routing Protocol for VANET
}

\author{
https://doi.org/10.3991/ijim.v15i17.24083
}

\author{
Safae Smiri( ${ }^{\bowtie)}$, Adil Ben Abbou, Abdelali Boushaba, \\ Azeddine Zahi, Rachid Ben Abbou \\ Sidi Mohamed Ben Abdellah University, Fez, Morocco \\ safae.smiri@usmba.ac.ma
}

\begin{abstract}
The extremely fast topology has created new requirements for the geographic routing protocol, which has been the most efficient solution for Vehicular Ad-hoc Networks (VANETs). The frequent disconnection of links makes the choice of the next routing node extremely difficult. Hence, an efficient routing algorithm needs to deliver the appropriate path to transfer the data packets with the most relevant quality of service (QoS). In this work, the weightaware greedy perimeter stateless (WA-GPSR) routing protocol is presented. The enhanced GPSR protocol computes the reliable communication area and selects the next forwarding vehicle based on several routing criteria. The proposal has been evaluated and compared to Maxduration-Minangle GPSR (MM-GPSR) and traditional GPSR using strict metric analysis. Our experimental results using NS-2 and VanetMobiSim, have demonstrated that WA-GPSR has the ability to enhance network performance.
\end{abstract}

Keywords - VANETs, geographical routing protocols, GPSR, QoS, VanetMobiSim, NS-2

\section{Introduction}

Over the recent decade, Vehicular Ad hoc Network (VANET) underwent tremendous technological advances. It is an integral part of the Intelligent Transportation System (ITS) [1]. VANETs provide many applications [2], such as board active safety, commercial services. Due to the fast mobility, the routing path is often happens reconstructed or broken when a vehicle transmits packets. Hence, the communication performance is affected in many situation according to speed, link lifetime, location of vehicles [3], etc. Thus, the research on VANET becomes crucial. In consequence, routing in VANETs has been a challenge that has led designers to try various strategies to solve it. To this purpose, routing design requires vigilance and development in VANET. Where, routing focuses on transmitting information from the source to the destination through an adequate path to enhance the travel convenience applications.

The classification of the routing protocols is according to two types: routing based on topology and geographic routing. The literature attests that geographical routing 
tends to be the most forceful and attractive one [4] [5]. Researchers in [6] attests that Geo-Networking has many merits and features, such as high scalability, good performance when velocity of vehcles is high and various information can be included in the status information like geographic location, time stamp, speed and exchange it via location based service. Greedy Perimeter StatelessRouting(GPSR) [7] is the most widely recognized geographical protocol, which exploits the geographic information of vehicles according to VANET characteristics. GPSR uses two routing mechanisms, the primary one is greedy routing which takes the closest vehicle to the destination as the next forwarding vehicle. The second is the perimeter transferwhich is invoked only when the greedy process is unsuccessful.

The proposed algorithm selects the most convenient vehicle for the next jump based on additional criteria and not only on the closest distance to the destination. The WA-GPSR considers various routing measures and assigns weights through additional features in order to offer a superior quality of service (QoS). In this work, we evaluate and compare our improved WA-GPSR protocol with the original GPSR protocol and the Maxduration-Minangle (MM-GPSR) GPSR protocol [8] using exhaustive QoS metrics. From the experimental results, WA-GPSR demonstrates improved performance in urban area.

The remaining section of this work is divided to the following structure: Section 2 discusses some related work. Section 3 presents the concept of a routing protocol based on the GPSR. Section 4 presents the proposed protocol. We present the experimental results with a discussion in section 5 . The conclusion is given in section 6 .

\section{Related work}

In VANET, the greater mobility and higher speed of vehicles lead to frequent update of routing table and irregular distribution of vehicles. Some of the studies conducted on the typical GPSR protocol are described below:

The authors of [9] proposed an another geographic routing protocol GpsrJ+ that improves GPCR by predicting an intuitive schema at the intersections. They use multiple metrics such as the speed and the direction to enhance the greedy forwarding strategy. The authors of [10] presented Multi-metric Geographic Routing (MGEDIR) for nexthop vehicle selection in urban area considering signal strength, distance to destination and direction. In a similar way, P-GPSR protocol [11] aims to select adequate relay vehicle by exploiting additional information like link stability, vehicle's speed and direction. The proposal presented as a multimetric geographic routing protocol (AMGRP) [12] adopts a hierarchical analytical AHP process based on link lifetime, nodes' density. The authors in [13] propose Adaptive GPSR protocol (AGPSR) that include additional information to select the best candidate node to forward packets by considering new field on Neighbors Table. The work [8] proposes Maxduration-Minangle GPSR which defines the allowed communication area in the greedy forwarding and selects the nodes with maximum cumulative communication as next-hop. In perimeter forwarding mode, they use the strategy of minimum angle to select the best next-hop node. The proposal 
work in [14] presented as Routing Based on Greedy Forwarding (GFR) selects the nexthop on the basis of the link quality and the distance between neighboring nodes. The authors define the quantity of neighbor nodes, which we cannot know the exact number of nodes from the source to the destination node.

Despite decades of research, we did not find a significant number of works that meet several routing metrics based on the reliable communication area, weight of all neighboring vehicles to improve the accident controlling applications with best QoS in VANETs. There is a necessity for more generalized algorithm to handle any network scenario. In this work, we propose an improved routing protocol called Weight-Aware Geographic Perimeter Stateless Routing protocol (WA-GPSR) based on additional features. We focus our work on urban scenarios where routing information is more complex due to the presence of intersections, building and traffic lights. The improved WA-GPSR protocol takes into account different routing measures and assigns weights obtained from a large number of parameter configurations. We have compared the performance of WA-GPSR with a recent proposal called MM-GPSR and the original GPSR protocol. Analyzing performance metrics, our results show that our improved WA-GPSR improves packets delivery ratio, reduces end-to-end delay and reached lowers routing cost as well as improves network efficiency.

\section{$3 \quad$ Location based routing protocol}

\subsection{Routing strategy}

GPSR is one of the most robust protocol to validate the concept of location-based routing strategy. Depending on the situation of communicating nodes, GPSR protocol uses two forwarding schemes for forwarding packets (see Figure 1(a)). It is assumed that every vehicle obtains its own position coordinates information by using positioning system such as GPS, GNSS [15].

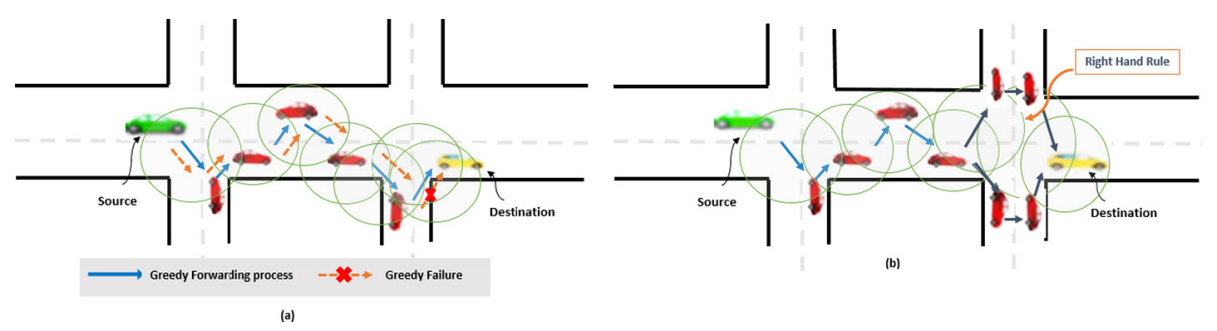

Fig. 1. Representation of GPSR functionality: (a) greedy process (b) perimeter Process

The greedy forwarding (GF) strategy is used to elect the best neighbor that is nearest to the destination. Nevertheless, if there is not a better neighbor nearest toward the destination. The packet will be routed through the perimeter routing process as shown in Figure 1(b) [7],[16],[17]. 


\subsection{The problem description}

In order to select the best neighbor in the GF strategy, the geographical location of the destination and neighbors is taken into account.

Generally, due to the fast changes of nodes, the node under selection as the best nexthop may be out of communication range. And therefore, the node may not receive the packet like Figure 2. In GF strategy, the current node (source node) selects node B as the next-hop node, however, the position of B, S, A moves to B', S', A', respectively. and then the node could not receive the packet because it is located not within the transmission range. Furthermore, the communication link is broken, which degrades the performance in the network.

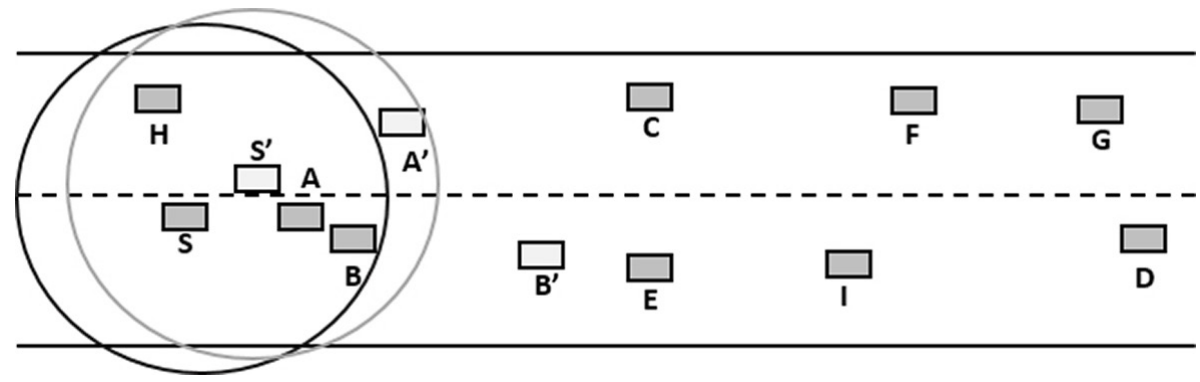

Fig. 2. The instability of the next-hop in the greedy transfer

\section{WA-GPSR: Weight-aware GPSR}

\subsection{Efficient communication area}

As depicted on Figure 3, S, A, B, H represent the nodes of the vehicle and the destination node is represented by $\mathrm{D}$. S will choose the closest node to the destination node from the neighboring nodes of the vehicle. The closest node to $\mathrm{D}$ is $\mathrm{B}(\mathrm{xB}, \mathrm{yB})$. The following is the distance between B and D (1), and between B and S (2):

$$
\begin{aligned}
& \mathrm{d}_{\mathrm{BD}}=\sqrt{\left(\mathrm{x}_{D}-\mathrm{x}_{B}\right)^{2}+\left(\mathrm{y}_{D}-\mathrm{y}_{B}\right)^{2}} \\
& \mathrm{~d}_{\mathrm{BS}}=\sqrt{\left(\mathrm{x}_{S}-\mathrm{x}_{B}\right)^{2}+\left(\mathrm{y}_{S}-\mathrm{y}_{B}\right)^{2}}
\end{aligned}
$$

The area where two circles overlap with the source $\mathrm{S}$ as center, $\mathrm{R}$ as radius, dmax as radius and is defined as the effective communication area. The blue part represents this area called ECA. The distance dmax is calculated by the subsequent formula (3) [8].

$$
\mathrm{d}_{\max }=\mathrm{d}_{\mathrm{BD}}+\lambda * \mathrm{~d}_{\mathrm{BS}}
$$


In the above equation (3), $\lambda \in[0,1]$. It appears that $\lambda$ affects the size of the area ECA. When $\lambda$ approaches 0 , ECA becomes smaller, subsequently the next-hop in the ECA area is easily selected as the node close to D. When $\lambda$ approaches 1 , ECA becomes longer and the next hop in ECA is selected as the node near S, however the node D may have an increase in the number of hops. When $\lambda$ is equal to 0.3 , it has a satisfactory performance in the greedy forwarding strategy.

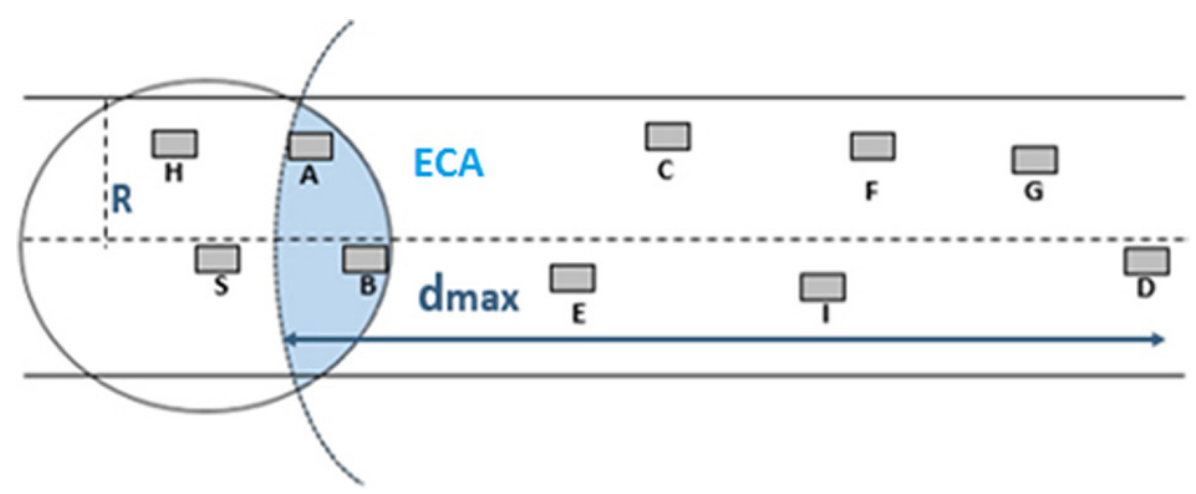

Fig. 3. Diagram of the efficient communication area

\subsection{Exchanging HELLO message and creating neighbor table}

In WA-GPSR, Hello messages are extended (as shown in Table 1) and used to exchange the required information by broadcasting it to neighboring nodes by a onehop communication. After a Hello message is received (Figure 4), a routing record is created in the neighbor table for every vehicles. Upon reception of the beacon packets, each source computes the average movement speed, mobility, link lifetime and cumulative time of the communication and stores them with the remaining neighbor information. Finally, when it receives Hello messages, the neighbor tables are updated and the routing measurements is used to calculate the weight of nodes. Thereafter, the packet is transmitted to the vehicle with highest weight.

Table 1. The format of HELLO packet in WA-GPSR

\begin{tabular}{|l|l|}
\hline \multicolumn{1}{|c|}{ Field Information } & \multicolumn{1}{c|}{ Description } \\
\hline ID & The unique node identifier \\
\hline Node Position $\left(\mathrm{X}_{-}, \mathrm{Y}_{-}\right)$ & The position coordinates $(\mathrm{X}, \mathrm{Y})$ of the vehicle \\
\hline Time Stamp $\left(\mathrm{t}_{-}\right)$ & The current time \\
\hline Speed & Moving speed of the vehicle $(\mathrm{m} / \mathrm{s})$ \\
\hline Num Neighbor & Number of neighbors of neighbor node \\
\hline Seqno_ & Sequence number of hello messages \\
\hline
\end{tabular}




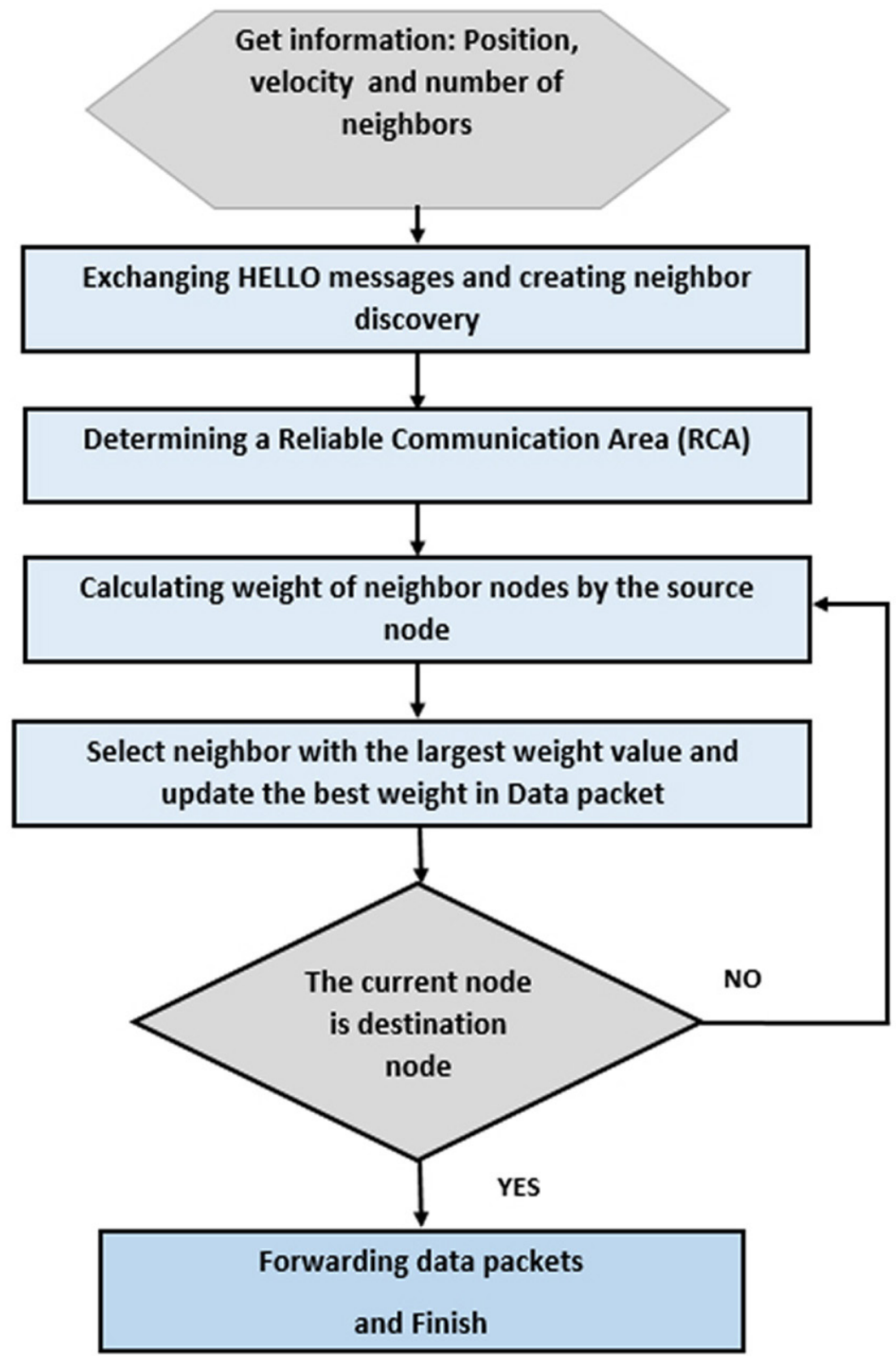

Fig. 4. Flowchart of WA-GPSR routing protocol

\subsection{Routing metrics}

The link lifetime. The smallest duration between nodes defines the link lifetime that remain in communication to transfer packets. Because of the variation in speed and different obstacles, the topology of the link changes and the nodes frequently break. 
Consider (xi, yi), (xs, ys) as the coordinates of neighbor i and source node $\mathrm{S}$ and the corresponding speeds given by vi, and vs (vs $<$ vi). Then, consider R as the communication range. Subsequently, the lifetime is solved according to Eq (4) [12]:

$$
L L T s, i=R-\frac{\sqrt{\left(\mathrm{x}_{i}-\mathrm{x}_{s}\right)^{2}+\left(\mathrm{y}_{i}-\mathrm{y}_{s}\right)^{2}}}{\mathrm{v}_{s}-\mathrm{v}_{i}}
$$

The cumulative communication time. The cumulative communication time $T_{i}$ builds a stable path and avoids frequent rebuilding of routing due to changing node positions in greedy mode. $T_{i}$ is calculated as follows [8]:

$$
\mathrm{T}_{\mathrm{i}}(\mathrm{t})=\mathrm{T}_{\mathrm{i}-1}+\Delta \mathrm{t}
$$

Where $\Delta \mathrm{t}=\mathrm{t}_{\mathrm{i}}-\mathrm{t}_{\mathrm{i}-1}$

In equation (5), $\mathrm{T}_{\mathrm{i}}-1$ is the last cumulative time of the reception of Hello message, ti is the current reception time of the Hello message. The cumulative time is initialized firstly at $\mathrm{T}_{1}=0$, by comparing $\mathrm{T}_{\mathrm{i}}$ in the area ECA (Figure 3 ), the node with maximum $\mathrm{T}_{\mathrm{i}}$ is chosen as the next-hop node of the source $\mathrm{S}$ (the node is steady to $\mathrm{S}$ and close to $\mathrm{D}$ ).

The traffic node density. Traffic is complex in many roads and the density of nodes is an important metric to determine the efficient routing path. The forward node having highest traffic density is chosen is computed as follow [18]:

$$
\text { Density }_{v}=\frac{\mathrm{N}_{\text {neighb }}}{R}
$$

Where $\mathrm{N}$ neighb designates the number of neighbours. $\mathrm{R}$ denotes the transmission range of the vehicle. The source path in the GPSR protocol is interrupted when there is no neighbor in the vicinity of the sending vehicle to transmit the packet. While our proposal protocol WA-GPSR establishes a reliable routing path by regarding the density of the traffic node.

The node's mobility. With a minimum mobility value, the mobility of vehicles s and $\mathrm{v}$ will be close to each other and they will stay longer in each other's communication area. Node mobility is computed using the follows formula [19]:

$$
\text { Mobility }_{\mathrm{v}}=\mid \operatorname{speed}_{\mathrm{s}}-\text { speed }_{\mathrm{v}} \mid
$$

Where, speeds and speed ${ }_{v}$ are the speeds of nodes s and v, respectively. By choosing a minimum value of Mobility, the mobility of vehicles $s$ and $v$ becomes closer and thus they will stay longer in the communication area of each other.

\subsection{Calculating of the weight of neighboring nodes}

The computation of the weight of the neighbor node proposed in the WA-GPSR routing protocol is calculated with the formula (8):

$$
\text { Weight }_{\mathrm{v}}=\mathrm{w}_{1} \times \operatorname{LLT}_{\mathrm{s}, \mathrm{i}}+\mathrm{w}_{2} \times \mathrm{T}_{\mathrm{i}}+\mathrm{w}_{3} \times \text { Density }_{\mathrm{v}}+\mathrm{w}_{4} \times\left(1 / \text { Mobility }_{\mathrm{v}}\right)
$$

Where $\left|\mathrm{w}_{1}\right|+\left|\mathrm{w}_{2}\right|+\left|\mathrm{w}_{3}\right|+\left|\mathrm{w}_{4}\right|=1$ 
In the above equation, Weight ${ }_{v}$ denotes the weight of vehicle $v, \operatorname{LLT}_{\mathrm{s}, \mathrm{i}}$ denotes the link lifetime of connections between the sender node $\mathrm{s}$ and the neighbor node $\mathrm{i}, \mathrm{Ti}$ denotes the cumulative communication time of node, Density ${ }_{v}$ denotes the traffic density of node, Mobility denotes the mobility of node $\mathrm{v}$ and wi are constant coefficient.

The pseudo code of the WA-GPSR routing protocol is described in algorithm 1 .

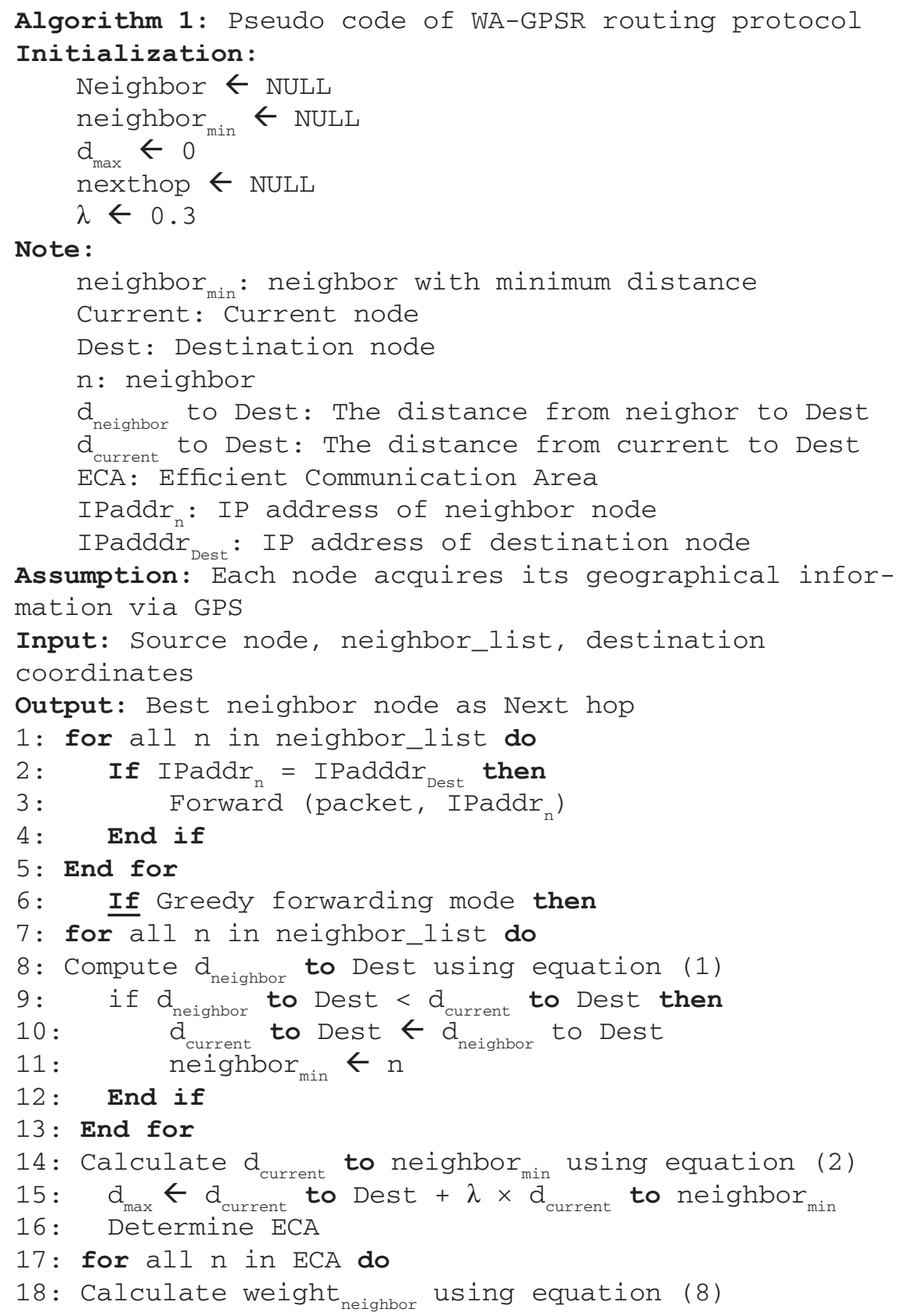




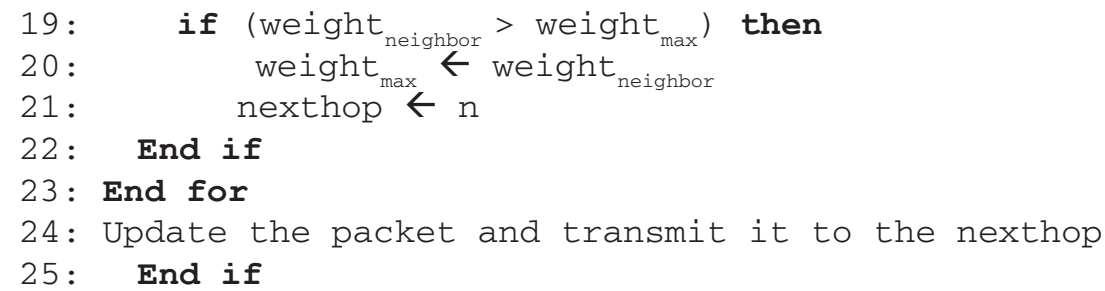

\section{$5 \quad$ Performance evaluation and results}

\subsection{The simulation setup}

The vehicle simulation has been conducted on an area of $1000 \mathrm{~m}^{2}$ and implemented in NS-2.35 [20]. Starting position of the vehicles used was random distribution. Table 2 presents the microscopic mobility model and Table 3 summarizes all additional parameters in our simulations.

Table 2. Parameters of the microscopic mobility model

\begin{tabular}{|l|l|c|}
\hline \multicolumn{1}{|c|}{ Parameter } & \multicolumn{1}{c|}{ Description } & Value \\
\hline $\mathrm{I}$ & Length of vehicles & $5 \mathrm{~m}$ \\
\hline A & Maximum acceleration & $0.6 \mathrm{~m} / \mathrm{s}^{2}$ \\
\hline B & Deceleration & $0.5 \mathrm{~m} / \mathrm{s}^{2}$ \\
\hline $\mathrm{A}_{\text {th }}$ & Threshold of acceleration & $0.2 \mathrm{~m} / \mathrm{s}^{2}$ \\
\hline So & Jam distance & $2 \mathrm{~m}$ \\
\hline T & Safety distance & $1.5 \mathrm{~s}$ \\
\hline P & Politeness & $0.5 \mathrm{~s}$ \\
\hline Mainstay & Min stay duration of node & $5 \mathrm{~s}$ \\
\hline Maxstay & Max stay duration of node & $30 \mathrm{~s}$ \\
\hline
\end{tabular}

Table 3. Simulation parameters

\begin{tabular}{|l|l|l|}
\hline \multicolumn{1}{|c|}{ Description } & \multicolumn{1}{c|}{ Value } & \multicolumn{1}{c|}{ Unit } \\
\hline Physical and link layer & & $\mathrm{m}$ \\
\hline Transmission range & 250 & - \\
\hline MAC protocol & IEEE802.11p & - \\
\hline Propagation model & Two-Ray-Ground & $\mathrm{s}$ \\
\hline Simulation time & 300 & \\
\hline Traffic model & & - \\
\hline Background traffic & CBR & connections \\
\hline CBR traffic connection & $5-10-15-20-25-30-35-40$ & packets/second \\
\hline Traffic packet rate & 8 & \\
\hline
\end{tabular}

(Continued) 
Table 3. Simulation parameters (continued)

\begin{tabular}{|l|l|l|}
\hline \multicolumn{1}{|c|}{ Description } & \multicolumn{1}{c|}{ Value } & \multicolumn{1}{c|}{ Unit } \\
\hline Traffic packet size & 512 & bytes \\
\hline Traffic direction & Multidirectional & \\
\hline Mobility model & & \\
\hline Simulation area size & $1000 \times 1000$ & $\mathrm{~m}^{2}$ \\
\hline Density node & $20-30-40-50-60-70-80-90-100$ & - \\
\hline Mobility model & IDM-LC & - \\
\hline IDM-LC model & & \\
\hline Vehicles speeds & 20 to 100 & $\mathrm{~km} / \mathrm{h}$ \\
\hline Lane per direction & 2 & - \\
\hline Traffic light & 10 & $\mathrm{~s}$ \\
\hline
\end{tabular}

The performance measures employed in the simulation are :

Packets Delivery Ratio (PDR): is computed by the subsequent equation:

$$
P D R=\frac{\text { Total number of received data packet }}{\text { Total number of sent data packet }}
$$

End-to-End Delay (E2ED): The amount of time that a packet spends on average moving from its source to its target. The subsequent equation is applied to compute the delay:

$$
E 2 E D=\frac{\text { Time of transmission }}{\text { Total number of received data packet }}
$$

Routing cost: is the ratio of the transmitted routing packets to number of data packet received at the destination. It is also a measure of the total number of control packets in the network. Its calculation is made by the following equation:

$$
\text { Routing cost }=\frac{\text { Total number of transmitted routing packet }}{\text { Total number of received data packet }}
$$

Network efficiency: defined as a ratio between the total number of data sent and the sum of the number of packets transmitted with the data sent. Its calculation is made by the following equation:

$$
\text { Network efficiency }=\frac{\sum \text { Number of sent data packet }}{\sum \text { Number of transmitted packet }+\sum \text { Number of data sent }}
$$

\subsection{Packets delivery ratio}

The packet delivery rate (PDR) of WA-GPSR, GPSR and MM-GPSR depicted in Figure 5(a) illustrates an increase in the number of CBR connections, especially, for WA-GPSR and MM-GPSR. This is because as more vehicles are connected to the 
network, the probability of having void problems is less. The simulation results illustrates that the proposal WA-GPSR protocol is more performant than MM-GPSR and GPSR, with a higher PDR for all results. Except for the results with 35 connections where MM-GPSR reaches its destination by the use of stability parameters leads to different route and then, higher PDR. For high CBR data traffic, MM-GPSR can help to balance the load on the network and then increase the PDR. Nevertheless, in that situation, the average difference between WA-GPSR and MM-GPSR is small $(<8 \%)$.
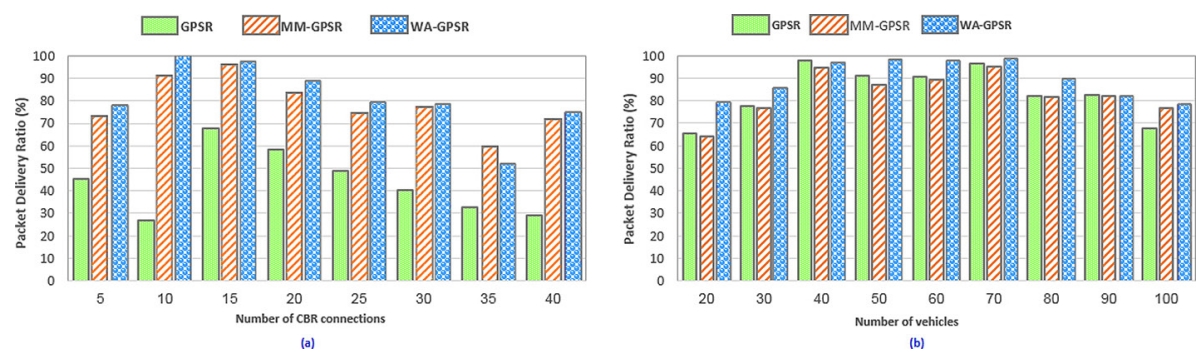

Fig. 5. Packets delivery ratio (a) as function of the number of $\mathrm{CBR}$ connection (b) as function of the number of vehicles

Compared to MM-GPSR and GPSR, WA-GPSR has a higher PDR on the overall results and aims to avoid communication disruptions. Figure 5(b) illustrates the comparison of PDRs involving WA-GPSR, GPSR and MM-GPSR at different numbers of vehicles. WA-GPSR surpasses GPSR and MM-GPSR due to having more source-destinations available in the urban scenario; the geographic routing protocols make it easy to locate the sending node. With a higher number of 70 vehicles, the WA-GPSR, MM-GPSR and GPSR protocols achieve a high PDR due to the short distance between nodes when the number of vehicles increases, resulting in a meaningful improvement in PDR. Overall, the WA-GPSR protocol has a higher PDR because it considers the stability of neighbouring nodes, link lifetime and density measurements to identify the optimum path to the destination.

\subsection{End to end delay}

Figure 6(a) displays the latency comparison of WA-GPSR, MM-GPSR and GPSR while making a different number of CBR connections. The latency increases of three implemented routing protocols. When transmitting packets, the neighbourhood relationship in urban environments is unreliable and unstable, resulting in higher path redundancy and therefore higher delay. On the whole, WA-GPSR has a clearly lower latency than MM-GPR and GPSR because of optimal routing path selection and a more stable next-hop node, resulting in a robust link of communication and hence a lower latency at the end. The packet will find the optimal path to its destination, resulting in a reduced delivery delay. In Figure 6(b), E2ED under our proposal appears to be smaller in comparison to MM-GPSR and GPSR, since our algorithm selects a more stable next sending node that will reduce the chance of missing vehicles in the communication range, resulting to a smaller E2ED. 

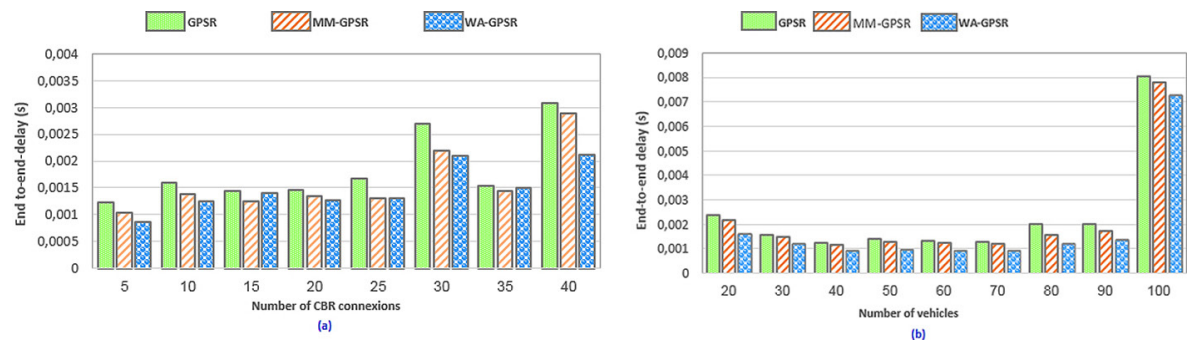

Fig. 6. E2ED (a) as function of the number of CBR connection (b) as function of the number of vehicles

\subsection{Routing cost}

The routing cost in Figure 7(a) decrease with the increase of CBR in the network. In some number of CBR connections. we have obtained a lower number of control packets of WA-GPSR which leads to a decrease in the routing cost. Nevertheless, the routing cost of WA-GPSR at 15, 30 and 35 connections is higher than MM-GPSR protocol by $2.9 \%, 7.4 \%$ and $3.3 \%$, respectively. Due to additional fields in Hello packets, leading the nodes to continuously update their one-hop neighbors with more information parameters. However, in low data traffic of about 5 to 10 connections, the routing cost of the GPSR is greater than the other two routing protocols. In most cases, the routing cost of WA-GPSR is lower than that of MM-GPSR and GPSR, mainly because WA-GPSR establishes a reasonable link that improves communication performance. Figure 7(b) depicts the routing cost of WA-GPSR, MM-GPSR and GPSR as a function of the number of vehicles. The routing cost of simulated protocols becomes higher in most situations. In every case, the routing cost of proposed WA-GPSR is less as compared to MM-GPSR and GPSR.
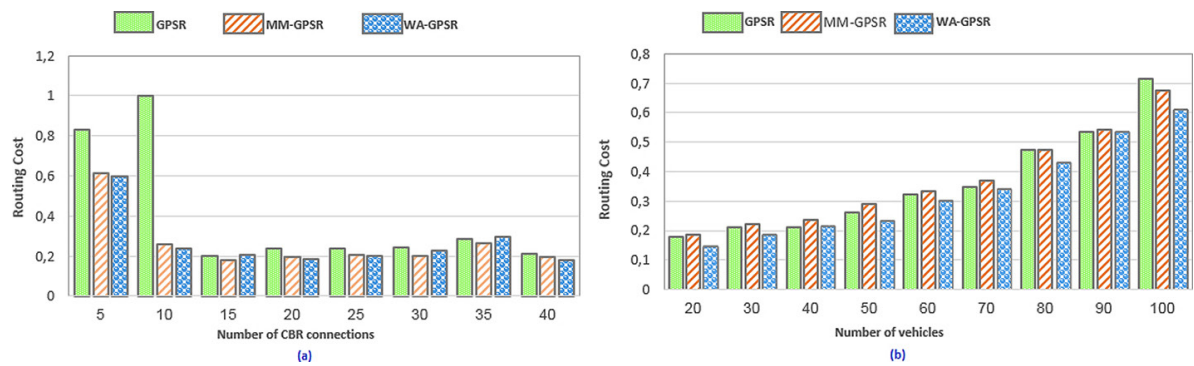

Fig. 7. Routing cost (a) as function of the number of CBR connection (b) as function of the number of vehicles

\subsection{Network efficiency}

Figure 8(a) illustrates the network efficiency that is important measure to indicate how well the data packets are efficiently delivered to the destination in the network. As the number of CBR connections rises, the network efficiency of WA-GPSR, 
MM-GPSR and GPSR improves, especially when the network is highly connected. The WA-GPSR protocol has a better performance in comparison to the other two routing protocols for almost all numbers of CBR connections. Indeed, as they are very appropriate in this scenario, they will make the packet to look for the most appropriate route toward the destination and subsequently increase the efficiency of the network. The exception is when the network is well connected at 30 and 35 connections; MM-GPSR has a higher efficiency. This can be explained by the recovery mode of MM-GPSR, which uses both the minimum angle and the right hand rule, which leads to the best path option in this situation. Figure 8(b) represents the network efficiency of WA-GPSR, MM-GPSR and GPSR according to the number of vehicles. The network efficiency declines as the number of vehicles increases. This is a consequence of the high rate of communication link failures as vehicle density increases. In general, the network efficiency of the WA-GPSR network is higher than that of MM-GPSR and GPSR, as shown in Figures 6(b) and 7(b), our WA-GPSR algorithm reduces the packet delivery time and controls the packets to achieve their destination, which also increases network efficiency.
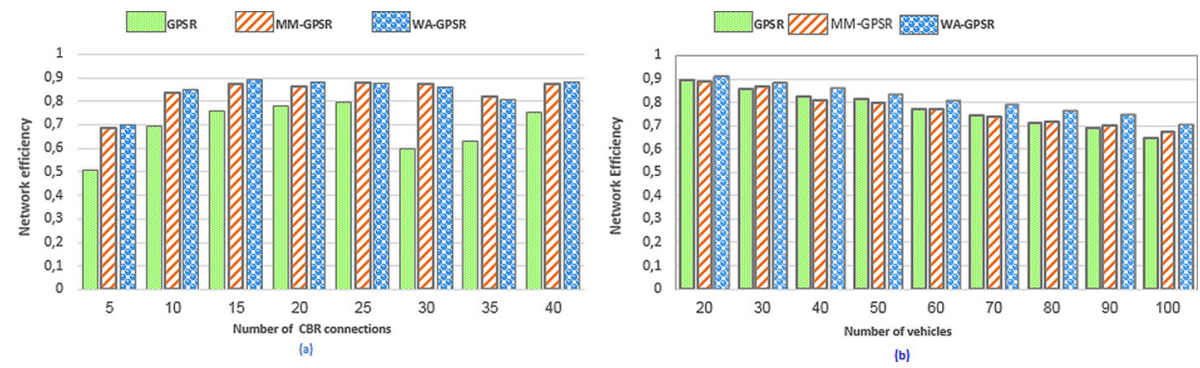

Fig. 8. Network efficiency (a) as function of the number of CBR connection (b) as function of the number of vehicles

\section{Conclusion}

The extremely dynamic topology and unpredictable behavior of VANET make routing design very difficult. In the presently work, we propose the WA-GPSR routing protocol which is based on the existing GPSR protocol, by discovering and exploiting neighbor information to obtain a steadier routing path. Our improved WA-GPSR optimizes and improves the greedy routing strategy, which is established in a reliable communication area and takes into account various routing measures to adapt the situation of link instability and find a better routing path. The experiments show that the performance of the enhanced protocol WA-GPSR outperforms that of MM-GPSR and GPSR in regards to packet delivery ratio, end-to-end delay, network efficiency and routing cost. 


\section{$7 \quad$ References}

[1] C. Campolo, A. Molinaro, and R. Scopigno,"Vehicular ad hoc Networks: Standards, Solutions, and Research". Springer, 2015. https://doi.org/10.1007/978-3-319-15497-8

[2] RAGAB, Ahmed Refaat, “A New Classification for Ad-Hoc Network” . iJIM, 2020, vol. 14, no 14, p. 215. https://doi.org/10.3991/ijim.v14i14.14871

[3] ALNABHAN, Mohammad, "Advanced GPSR in Mobile Ad-hoc Networks (MANETs)". 2020. https://doi.org/10.3991/ijim.v14i18.16661

[4] S. Smiri, A. Boushaba, R. B. Abou, and A. Zahi, "Geographic and topology based routing protocols in vehicular adhoc networks: Performance evaluation and QoS analysis", in 2018 International Conference on Inteligent Systems and Computer Vision (ISCV), Apr. 2018, pp. 1-8. https://doi.org/10.1109/ISACV.2018.8354070

[5] HOUMER, Meriem, OUAISSA, Mariya, OUAISA, Mariyam, et al. SE-GSPR: Secured and Enhanced Greedy Perimeter Stateless Routing Protocol for Vehicular Ad hoc Networks. 2020. https://doi.org/10.3991/ijim.v14i13.14537

[6] Marfia, Gustavo, "Safe driving in LA: Report from the greatest intervehicular accident detection test ever", IEEE Transactions 62.2 (2012). https://doi.org/10.1109/TVT.2012.2226484

[7] B. Karp and H. T. Kung, "GPSR: Greedy Perimeter Stateless Routing for Wireless Networks", in Proceedings of the 6th Annual InternationalConferenceonMobileComputing and Networking, New York, NY, USA, 2000, pp. 243-254. https://doi.org/10.1145/345910.345953

[8] X. Yang, M. Li, and T. Di, "Improvement of GPSR Protocol in Vehicular Ad Hoc Network", IEEE Access, vol. 6, pp. 39515-39524, 2018. https://doi.org/10.1109/ ACCESS.2018.2853112

[9] K. C. Lee, J. Haerri, U. Lee, and M. Gerla, "Enhanced PerimeterRouting for Geographic Forwarding Protocols in Urban VehicularScenarios", in 2007 IEEEGlobecomWorkshops, Washington, DC, USA, Nov. 2007, pp. 1-10. https://doi.org/10.1109/ GLOCOMW.2007.4437832

[10] A.N. Hassan, A. H. Abdullah, O. Kaiwartya, Y. Cao, and D. K. Sheet, "Multi-metric geographic routing for vehicular ad hoc networks", Wirel. Netw., vol. 24, no. 7, pp. 2763-2779, Oct. 2018. https://doi.org/10.1007/s11276-017-1502-5

[11] S. Dahmane and P. Lorenz, "Weighted Probabilistic Next-Hop Forwarder Decision-Making in VANETEnvironments", in 2016 IEEE Global CommunicationsConference (GLOBECOM), Washington, DC, USA, Dec. 2016, pp. 1-6. https://doi.org/10.1109/ GLOCOM.2016.7842381

[12] N. V. Dharani Kumari and B. S. Shylaja, "AMGRP: AHP-based Multimetric Geographical RoutingProtocolfor Urban environmentofVANETs", J. King Saud Univ. - Comput. Inf. Sci., vol. 31, no. 1, pp. 72-81, Jan. 2019. https://doi.org/10.1016/j.jksuci.2017.01.001

[13] A. Silva, K. M. Niaz Reza, and A. Oliveira, "An Adaptive GPSR Routing Protocol for VANETs", in 15th InternationalSymposium on WirelessCommunicationSystems (ISWCS), Lisbon, Aug. 2018, pp. 1-6. https://doi.org/10.1109/ISWCS.2018.8491075

[14] X. Yang, M. Li, Z. Qian, and T. Di, "Improvement of GPSR Protocol in Vehicular Ad Hoc Network,” IEEEAccess, vol. 6, pp. 39515-39524, 2018. https://doi.org/10.1109/ ACCESS.2018.2853112

[15] Zhang, De-Gan, et al. "A new method of mobile ad hoc networkrouting based ongreed forwarding improvement strategy". IEEEAccess 7 (2019). https://doi.org/10.1109/ ACCESS.2019.2950266

[16] J. Liu, J. Wan, Q. Wang, P. Deng, K. Zhou, and Y. Qiao, “A survey on position-based routing for vehicular ad hoc networks”, Telecommun. Syst., vol. 62, no. 1, pp. 15-30, May 2016. https://doi.org/10.1007/s11235-015-9979-7 
[17] S. Smiri et al., "Performance Analysis of Routing Protocols with Roadside Unit Infrastructure in a Vehicular AdHocNetwork", Int. J. Comput. Netw. Commun. IJCNC, vol. 12, no. 4, pp. 19, Jul. 2020. https://doi.org/10.5121/ijenc.2020.12402

[18] Wang, Xi, and Feifi Li. "Wirless Sensor Networks Suitable for Large-Scale Heterogeneous Networking." International Journal of Online \& Biomedical Enginering 15.1,2019. https:// doi.org/10.3991/ijoe.v15i01.9779

[19] Arianmehr, Sharbanou, and Ali. "HybTGR: a hybrid routing protocol based on topological and geographical information in vehicular ad hoc networks". Journal of Ambient IntelligenceandHumanizedComputing (2019), pp. 1-13. https://doi.org/10.1007/s12652-019-01332-Z

[20] T. Issariyakul and E. Hossain, "Introduction to Network Simulator 2 (NS2)", in Introduction to NetworkSimulatorNS2. Boston, MA: Springer US, 2012, pp. 21-40. https://doi. org/10.1007/978-1-4614-1406-3_2

\section{Authors}

Safae Smiri received her master's degree from faculty of Sciences and Techniques of Fez in 2016. Currently, she is a Ph.D Student at SIA-Laboratory at the Faculty of Science and Technology of Fez. Her research interests include Computer Networking, Telecommunications, Routing protocols, Communication Technology and Quality of Service. Email: safae.smiri@usmba.ac.ma

Adil Ben Abbou is a Research Professor in Faculty of Science and Technology, Sidi Mohamed Ben Abbdellah university of Fez. He is also a member of SIA-Laboratory. His main research interest is: Network Communication, Performance evaluation, Quality of Service, Optimisation and Topology Control Traffic in Ad hoc Networks.

Abdelali Boushaba is a Professor in Computer Sciences at Faculty of Science and Technology, Sidi Mohamed Ben Abdellah university of Fez. His research interests include Performance Evaluation, Quality of Service, Quality of Experiment, Multimedia Communications, Optimization and Fuzzy Logic Applications in Wireless Ad hoc Networks.

Azeddine Zahi received his PhD degree in 1997 in Computer Sciences from Mohammed V University in Rabat. He is currently a research professor at Sidi Mohamed Ben Abbdellah University of Fez since 1995. He is also a member of SIA-Laboratory. His research interests include data mining, artificial intelligence and Ad hoc Network.

Rachid Ben Abbou is a Professor at Sidi Mohamed Ben Abbdellah University in Fez since 1997. Former Head of computer science department at Faculty of Science and Technology. Member of SIA-Laboratory. His research interest concern ad hoc network, security in Cloud computing, e-learning, Automatic Assessment.

Article submitted 2021-05-18. Resubmitted 2021-06-20. Final acceptance 2021-06-20. Final version published as submitted by the authors. 